\title{
Strengthening Your Hip Muscles: Some Exercises May Be Better Than Others
}

\author{
Amanda Ulin, Kelsey Draper, Kaylee Draper \\ Departmental Affiliation: Kinesiology \\ College of Arts and Sciences
}

Weak hip muscles lead to poor hip motion, and poor hip motion can cause knee, hip, and back pain. Strengthening hip muscles that control how hips move may reduce pain in these parts of the body. This undergraduate research project compared hip abductor muscle activity during selected exercises using electromyography in order to determine which exercises are best for activating the gluteus medius and the superior portion of the gluteus maximus, while minimizing activity of the tensor fascia lata. Participants in this study included nine healthy female soccer players between the ages of 18 and 21. Using an electromyogram (EMG), the muscle activity of the nine subjects was recorded during the following 11 exercises: hip abduction in sidelying, clam with elastic resistance around thighs, bilateral bridge, unilateral bridge, hip extension in quadruped on elbows with knee extending, hip extension in quadruped on elbows with knee flexed, forward lunge with erect trunk, squat, sidestep with elastic resistance in a squatted position, hip hike, and forward step-up. At this time, data collection and analysis continues; however, it is hypothesized that both gluteal muscles were significantly more active than the TFL in unilateral and bilateral bridging, quadruped hip extension, the clam, sidestepping, and squatting.

\section{Information about the Authors:}

Amanda Ulin is a senior from Solon, Iowa, majoring in biology and minoring in exercise science and psychology. She will pursue a doctorate degree in physical therapy at the University of Iowa this fall. Kelsey and Kaylee Draper are juniors from Perrysburg, Ohio. They are both majoring in biology with minors in chemistry and psychology. After graduating in May 2015, they plan to pursue a master's degree in physician assistant studies.

\section{Faculty Sponsor: Dr. Kelly Helm}

Student Contact: amanda.ulin@valpo.edu 